\title{
Selections and near-selections in metric linear spaces without local convexity
}

\author{
by \\ Tadeusz Dobrowolski (Pittsburg, KS) and Jan van Mill (Amsterdam)
}

\begin{abstract}
We characterize the AR property in convex subsets of metric linear spaces in terms of certain near-selections.
\end{abstract}

1. Introduction. All spaces under discussion are metrizable.

As is well known, every locally convex metric linear space is an Absolute Retract (abbreviated AR; Dugundji [18]). (Since we restrict ourselves to metrizable spaces, every AR is an Absolute Extensor, i.e., a space that can play the role of $\mathbb{R}$ in the classical Tietze-Urysohn Theorem, and vice versa.) The local convexity assumption in this result is not essential, as the $\ell^{p}$-spaces $(0<p<1)$ show. Other examples can be found within the class of $\aleph_{0}$-dimensional metric linear spaces, i.e., metric linear spaces having a countable Hamel basis. This follows from Haver's Theorem in [20] that every locally contractible space that is a countable union of finite-dimensional compacta is an Absolute Neighborhood Retract (abbreviated: ANR). The formidable problem of whether every metric linear space is an AR was finally settled by Cauty [9] with a negative answer. Detecting the AR-property in infinite-dimensional metric linear spaces and topological groups is usually the first step towards proving that they are, in fact, homeomorphic to an infinite-dimensional manifold (see, e.g., Dobrowolski and Toruńczyk [17]). But even for a space as concrete as the group of all homeomorphisms of the 3-cell, it is unknown whether it is an AR.

The usual and well known proof of the fact that every locally convex metric linear space is an AR, is by applying the so-called Dugundji Extension

2000 Mathematics Subject Classification: 55M15, 54C60.

Key words and phrases: metric linear space, locally convex, compact convex set, selection, near-selection, finite-dimensional, $C$-space.

The first-named author gratefully acknowledges support from grant B 61-541 of the Nederlandse Organisatie voor Wetenschappelijk Onderzoek (NWO) during the summer of 2004. 
Formula [5, p. 57]. It seems less well known that this result can also be proved by a (near-)selection argument (cf. [5, p. 92], [26, 1.4.13], [28, p. 232]; see also the proof of Theorem 4.2 below). The aim of this paper is to characterize the AR-property in convex subsets of metric linear spaces in terms of certain near-selections. Roughly speaking, our characterization theorem states that a convex set in a metric linear space is an AR if and only if lower semicontinuous functions with finite-dimensional compact convex values in it admit near-selections.

For some of our results it suffices that the spaces under consideration are merely paracompact since that is the only thing used in the proofs. We do not aim at full generality and so our results are sometimes not stated in their most general form.

We are indebted to Ernie Michael, Pavel Semenov, Valentin Gutev and the referee for helpful comments.

2. Preliminaries. We adopt the usual terminology with respect to partitions of unity (Dugundji [19]). So, if $f: X \rightarrow \mathbb{R}$ is continuous, then the support of $f$ is the closed set $\{x \in X: f(x) \neq 0\}$. If $\left\{\kappa_{i}: i \in I\right\}$ is a partition of unity on a space $X$, then the supports of the $\kappa_{i}$ form a locally finite closed covering of $X$. Hence, in particular, for each $x \in X$ there are only finitely many $i \in I$ with $\kappa_{i}(x) \neq 0$. The following central result on partitions of unity will be used several times without explicit reference: for each open cover $\mathcal{U}=\left\{U_{i}: i \in I\right\}$ of a space $X$ there is a partition of unity $\left\{\kappa_{i}: i \in I\right\}$ on $X$ such that $\overline{\kappa_{i}^{-1}(0,1]} \subseteq U_{i}$ for every $i \in I$. Such a partition of unity is said to be subordinated to $\mathcal{U}$. See [19, p. 170] for details.

If $(X, d)$ is a space and $x \in X$, then $B(x, \varepsilon)$ (respectively, $B(A, \varepsilon)$ ) abbreviates the open ball about $x$ (respectively, about $A \subseteq X$ ) of radius $\varepsilon$.

Let $E$ be a metric linear space. We will always consider $E$ equipped with a translation invariant metric $d$ induced by a so-called $F$-norm $|\cdot|$ on $E$ (that is, $d(x, y)=|x-y|)$. We require that such a norm is increasing on each ray emanating from the origin (or, equivalently, $|t x| \leq|x|$ for all real numbers $t$ with $|t| \leq 1$ ). See, e.g., [29, Theorem 1.24] for details. A subset $X$ of $E$ is called complete if the metric $d$ restricted to $X$ is a complete metric. Clearly, every complete subset of $E$ is closed in $E$ and every compact subset of $E$ is complete. The zero vector of $E$ will be denoted by $\underline{0}$.

If $E$ is a metric linear space that is an AR, then so are all of its dense linear subspaces. This can be seen as follows. First one observes that if $L$ is a dense linear subspace of $E$ then $E \backslash L$ is locally homotopy negligible in $E$ (this is folklore, for a proof see, e.g., Dobrowolski and Mogilski [16, Lemma 3.1]). It then suffices to apply Toruńczyk's [32, Theorem 3.1]. The same can 
be said about dense convex sets of arbitrary convex sets in metric linear spaces. We will use this fact frequently without explicit reference.

3. Set-valued functions. Let $X$ and $Y$ be sets. By writing $F: X \Rightarrow Y$ we shall mean that $F$ is a set-valued function from $X$ to $Y$. That is, $F(x)$ is a nonempty subset of $Y$ for every $x \in X$.

Let $X$ and $Y$ be topological spaces and let $F: X \Rightarrow Y$. For every $V \subseteq Y$ we put

$$
F^{\Leftarrow}(V)=\{x \in X: F(x) \cap V \neq \emptyset\} .
$$

Then $F$ is lower semicontinuous (abbreviated LSC) provided that for every open subset $U$ of $Y, F^{\Leftarrow}(U)$ is open in $X$. In addition, a (continuous) selection for $F$ is a (continuous) function $f: X \rightarrow Y$ such that $f(x) \in F(x)$ for every $x \in X$.

REMARK 3.1. Let $E$ be a metric linear space. In addition, let $F: X \Rightarrow E$ be a function such that for every $x \in X, F(x)$ is convex. Assume that $X$ has an open cover $\mathcal{U}$ such that for every $U \in \mathcal{U}$ the function $F \mid U$ has a continuous selection, say $f_{U}$. Hence continuous selections for $F$ exist locally. We claim that in this situation $F$ has in fact a global continuous selection. Let $\left\{\kappa_{U}\right\}_{U \in \mathcal{U}}$ be a partition of unity subordinated to $\mathcal{U}$. Then the function $f$ defined by the formula

$$
f(x)=\sum\left\{\kappa_{U}(x) f_{U}(x): U \in \mathcal{U}, x \in U\right\}
$$

is a continuous selection for $F$. This useful observation, perhaps due to Corson and Lindenstrauss [11, proof of Theorem 2.1], will be used without explicit reference below.

REMARK 3.2. The famous Michael Selection Theorem from [23] asserts that if $F: X \Rightarrow E$ is LSC, where $E$ is a locally convex metric linear space and every $F(x)$ is both complete and convex, then $F$ admits a continuous selection. Continuous selections are important tools in topology and functional analysis. There are several versions of the Michael Selection Theorem which all assume local convexity in one form or another. We could not find an example in the literature of a metric linear space for which the conclusion of the Michael Selection Theorem fails. Let us present such an example. By Cauty [9], there is a metric linear space $Z$ that is not an AR. By passing to the completion of $Z$, we may assume that the Cauty space $Z$ is complete. We may assume that $Z$ is a closed subspace of some AR $X$ (see [26, Lemma 1.2.3]). Set $F(x)=\{x\}$ for $x \in Z$ and $F(x)=Z$ for $x \notin Z$. Then $F: X \Rightarrow Z$ is LSC and does not have a continuous selection, since otherwise $Z$ would be a retract of $X$.

We now formulate and prove four simple lemmas. 
Lemma 3.3. Let $E$ be a metric linear space. In addition, let $F: X \Rightarrow E$ be an LSC-function such that for every $x \in X, F(x)$ is a finite-dimensional convex set. Then, for every $n \in \mathbb{N}, X_{n}=\{x \in X: \operatorname{dim} F(x) \leq n-1\}$ is closed in $X$.

Proof. We will show that $\{x \in X: \operatorname{dim} F(x) \geq n\}$ is open. Pick an arbitrary $x_{0}$ such that $\operatorname{dim} F\left(x_{0}\right) \geq n$. We may assume that $F\left(x_{0}\right)$ contains the origin. Then $F\left(x_{0}\right)$ contains $n$ linearly independent vectors $v_{1}, \ldots, v_{n}$. Take pairwise disjoint open balls $B_{i}=B\left(v_{i}, r_{i}\right)$ so that for every choice of $x_{i} \in B_{i}, i=1, \ldots, n$, the set $\left\{x_{1}, \ldots, x_{n}\right\}$ is linearly independent. Let $U=F^{\Leftarrow}\left(B_{1}\right) \cap \cdots \cap F^{\Leftarrow}\left(B_{n}\right)$, and observe that $U$ is an open neighborhood of $x_{0}$. Then, for every $x \in U$, we have $F(x) \cap B_{i} \neq \emptyset, i=1, \ldots, n$. Hence, $F(x)$ contains at least $n$ linearly independent vectors and, consequently, $\operatorname{dim} F(x) \geq n$.

Let $X$ and $(Y, d)$ be spaces. If $F: X \Rightarrow Y$ and $\varepsilon>0$, then a continuous function $f: X \rightarrow Y$ is called an $\varepsilon$-near-selection of $F$ if $d(f(x), F(x))<\varepsilon$ for every $x \in X$. (So a continuous selection can be thought of as a "0-nearselection" provided every $F(x)$ is closed in $Y$.) This is the central concept in this paper.

We are primarily interested here in $\varepsilon$-near-selections in the case that $Y$ is a convex subspace of a metric linear space $E, F$ has convex values, and $d$ is the metric on $Y$ we get from an arbitrary $F$-norm on $E$.

LemmA 3.4. Let $X$ and $(Y, d)$ be spaces, let $F: X \Rightarrow Y$ be LSC, and let $\varepsilon>0$. In addition, let $X_{0}$ be a subset of $X$ for which there exists a map $f: X \rightarrow Y$ such that $f \mid X_{0}$ is an $\varepsilon$-near-selection for $F \mid X_{0}$. Then there is a neighborhood $U_{\varepsilon}$ of $X_{0}$ such that $f \mid U_{\varepsilon}$ is a $2 \varepsilon$-near-selection of $F \mid U_{\varepsilon}$.

Proof. It is easy to see that

$$
U_{\varepsilon}=\bigcup_{x \in X_{0}} f^{-1}(B(f(x), \varepsilon)) \cap F^{\Leftarrow}(B(f(x), \varepsilon))
$$

is as required.

We now present a controlled version of Remark 3.1 for near-selections.

LeMma 3.5. Let $Y$ be a convex subspace of a metric linear space and let $F: X \Rightarrow Y$ be an LSC-function with convex values. Moreover, assume that $X$ can be written as $\bigcup_{n=1}^{\infty} U_{n}$ such that for every $n \in \mathbb{N}, U_{n}$ is open and $F \mid U_{n}$ admits an $\varepsilon_{n}$-near-selection $F_{n}: U_{n} \rightarrow Y$. Then there is a continuous function $f: X \rightarrow Y$ such that $d(f(x), F(x)) \leq \sum\left\{\varepsilon_{n}: x \in U_{n}\right\}$ for every $x \in X$.

Proof. Define $\bar{F}_{n}(x)$ for every $x \in X$ by letting $\bar{F}_{n}(x)=F_{n}(x)$ if $x \in U_{n}$, and $\bar{F}_{n}(x)=\underline{0}$ if $x \notin U_{n}$. 
For the open cover $\left\{U_{n}: n \in \mathbb{N}\right\}$ of $X$ we pick an arbitrary partition of unity $\left\{\lambda_{n}\right\}_{n \in \mathbb{N}}$ subordinated to it, and define the function $f: X \rightarrow Y$ by the formula $f(x)=\sum_{n=1}^{\infty} \lambda_{n}(x) \bar{F}_{n}(x)$.

It is clear that $f$ is well defined and continuous. Take an arbitrary $x \in X$, and let $A=\left\{n \in \mathbb{N}: \lambda_{n}(x)>0\right\}$. By construction, for every $n \in A$ there exists an element $z_{n} \in F(x)$ such that $\left|z_{n}-\bar{F}_{n}(x)\right|<\varepsilon_{n}$. Put $z=$ $\sum_{n \in A} \lambda_{n}(x) z_{n}$. Then $z \in F(x)$ since $F(x)$ is convex, and

$$
|f(x)-z| \leq \sum_{n \in A}\left|\lambda_{n}(x)\left(\bar{F}_{n}(x)-z_{n}\right)\right| \leq \sum_{n \in A}\left|\bar{F}_{n}(x)-z_{n}\right| \leq \sum_{n \in A} \varepsilon_{n}
$$

as required.

LEMMA 3.6. Let $Y$ be a convex subspace of a metric linear space E, and let $F: X \Rightarrow Y$ be an LSC-function with convex values. Then the following conditions are equivalent:

(1) $F$ admits for every $\varepsilon>0$ an $\varepsilon$-near-selection $f: X \rightarrow Y$.

(2) For every continuous function $\varepsilon: X \rightarrow(0,1)$ there exists a continuous function $f: X \rightarrow Y$ such that $d(f(x), F(x))<\varepsilon(x)$ for every $x \in X$.

Proof. For $(1) \Rightarrow(2)$, let $\varepsilon: X \rightarrow(0,1)$ be continuous, and for every $n \geq 0$, put

$$
D_{n}=\left\{x \in X: 2^{-(n+2)}<\varepsilon(x)<2^{-n}\right\} .
$$

In addition, for every $n \geq 0$ let $f_{n}: X \rightarrow Y$ be a $2^{-(n+3)}$-near-selection of $F$. There exists by Lemma 3.5 a continuous function $f: X \rightarrow Y$ such that $d(f(x), F(x)) \leq \sum\left\{2^{-(n+3)}: x \in D_{n}, n \in \mathbb{N}\right\}$ for every $x \in X$. To prove that $f$ is a near-selection for $F$ which is controlled by the function $\varepsilon$, take an arbitrary $x \in X$, and let $n(x)=\min \left\{n \geq 0: x \in D_{n}\right\}$. Observe that if $x \in D_{m}$, then $m \in\{n(x), n(x)+1\}$. Hence

as required.

$$
d(f(x), F(x)) \leq \frac{1}{2^{n(x)+3}}+\frac{1}{2^{n(x)+4}}<\frac{1}{2^{n(x)+2}}<\varepsilon(x),
$$

Since $(2) \Rightarrow(1)$ is trivial, this completes the proof.

4. Main results. In this section we will present our main results, Theorems 4.1 and 4.2. In Theorem 4.1 we show that certain LSC-maps have a continuous selection. This result is then used in Theorem 4.2 to prove our characterization of the AR-property in convex subsets of metric linear spaces in terms of near-selections.

In [25, Corollary 7.3], Michael proved that if $G$ is a topological group, and $H$ a closed subgroup isomorphic to the additive group of a Fréchet space, then the natural quotient map from $G$ to $G / H$ has a cross-section. For the special case that $G$ is a complete metric linear space, see [5, Prop. 7.1 on 
p. 87] (the proof presented there is due to Toruńczyk). Theorem 4.1 below and its proof are inspired by these results.

We will use the following elementary but useful fact due to Carathéodory. Let $E$ be an $n$-dimensional metric linear space. Then if $x$ lies in the convex hull of a set $F \subseteq E$, then $x$ lies in the convex hull of some subset of $F$ that contains at most $n+1$ points [30, p. 3]. This easily implies that for every $r>0$ the convex hull of the ball $B(\underline{0}, r)$ in $E$ is contained in $B(\underline{0},(n+1) r)$.

TheOREM 4.1. Let $E$ be a metric linear space E. Suppose that $F: X \Rightarrow E$ is an LSC-function with closed and convex values. If there is an open cover $\mathcal{U}$ of $X$ such that for every $U \in \mathcal{U}, \max \{\operatorname{dim} F(x): x \in U\}<\infty$, then $F$ admits a continuous selection.

Proof. We will first prove the theorem in the special case that $\mathcal{U}=\{X\}$.

Let $n \in \mathbb{N}$ be such that $\operatorname{dim} F(x) \leq n$ for all $x \in X$. By induction on $k \geq 0$ we will construct a sequence of (not necessarily continuous) functions $f_{k}: X \rightarrow E$ so that

(1) $f_{k}(x) \in F(x), x \in X$,

(2) for every $x_{0} \in X$ there exists a neighborhood $V_{x_{0}}^{k}$ of $x_{0}$ such that $\left|f_{k}(x)-f_{k}\left(x_{0}\right)\right| \leq 1 / 2^{k}$ whenever $x \in V_{x_{0}}^{k}$,

(3) $\left|f_{k}(x)-f_{k-1}(x)\right| \leq 1 / 2^{k}+(n+1) / 2^{k-1}, x \in X$.

Having constructed this sequence, we let $f$ denote its pointwise limit. Observe that for every $x \in X, f(x)$ is well defined and belongs to $F(x)$ since $F(x)$ being finite-dimensional and convex is complete with respect to any $F$-norm $|\cdot|$ on $E$. Moreover, for every $x \in V_{x_{0}}^{k}$,

$$
\begin{aligned}
\left|f(x)-f\left(x_{0}\right)\right| & \leq\left|f(x)-f_{k}(x)\right|+\left|f_{k}(x)-f_{k}\left(x_{0}\right)\right|+\left|f_{k}\left(x_{0}\right)-f\left(x_{0}\right)\right| \\
& \leq(n+1) / 2^{k-2}+3 / 2^{k} .
\end{aligned}
$$

This yields the continuity of $f$.

Assuming the $F$-norm $|\cdot|$ is bounded by 1 , for $k=0$, we see that condition (2) is satisfied with $V_{x_{0}}^{0}=X, x_{0} \in X$, and any selection $f_{0}: X \rightarrow E$ of $F$.

Suppose $f_{0}, \ldots, f_{k-1}$ have been constructed for some $k \geq 1$. Let $\delta>0$ be so small that $(2 n+1) \delta \leq 1 / 2^{k+2}$.

Consider for a fixed $x_{0} \in X$ its open neighborhood

$$
U_{x_{0}}=V_{x_{0}}^{k-1} \cap F^{\Leftarrow}\left(B\left(f_{k-1}\left(x_{0}\right), \delta\right)\right) .
$$

Since by (i), $F(x) \cap B\left(f_{k-1}\left(x_{0}\right), \delta\right) \neq \emptyset$ for every $x \in U_{x_{0}}$, there is a (possibly discontinuous) selection $g_{x_{0}}: U_{x_{0}} \rightarrow E$ for the function $F \mid U_{x_{0}}$ which, for every $x \in U_{x_{0}}$, satisfies

$$
\left|g_{x_{0}}(x)-f_{k-1}\left(x_{0}\right)\right|<\delta
$$

Let $g_{x_{0}} \mid\left(X \backslash U_{x_{0}}\right)$ be an arbitrary constant function. By (ii),

$$
\operatorname{diam} g_{x_{0}}\left(U_{x_{0}}\right)<2 \delta
$$


and by (i) and (2),

(iv) $\left|g_{x_{0}}(x)-f_{k-1}(x)\right| \leq \mid g_{x_{0}}(x)-f_{k-1}\left(x_{0}|+| f_{k-1}\left(x_{0}\right)-f_{k-1}(x) \mid \leq \delta+\frac{1}{2^{k-1}}\right.$ for every $x \in U_{x_{0}}$.

Let $\mathcal{P}=\left\{p_{x_{0}}: x_{0} \in X\right\}$ be a partition of unity subordinated to the open cover $\left\{U_{x_{0}}: x_{0} \in X\right\}$. Define $f_{k}: X \rightarrow E$ by the formula

$$
f_{k}(x)=\sum_{x_{0} \in X} p_{x_{0}}(x) g_{x_{0}}(x)
$$

and notice that (1) holds (here we use the convexity of $F(x)$ ).

Fix $x \in X$, and write $E(x)=\left\{x_{0} \in X: x \in p_{x_{0}}^{-1}(0,1]\right\}$ and $E^{\prime}(x)=$ $\left\{x_{0} \in X: x \in \overline{p_{x_{0}}^{-1}(0,1]}\right\}$; observe that $E(x) \subseteq E^{\prime}(x)$ and that $E^{\prime}(x)$ is finite. Clearly,

$$
\left|f_{k}(x)-f_{k-1}(x)\right|=\sum_{x_{0} \in E(x)} p_{x_{0}}(x)\left(g_{x_{0}}(x)-f_{k-1}(x)\right) .
$$

Since all differences $g_{x_{0}}(x)-f_{k-1}(x)$ for $x_{0} \in E(x)$ belong to an $n$-dimensional linear subspace of $E$ and are by (iv) of $F$-norm smaller than $r=$ $\delta+2^{-k+1}$, it follows by the remark preceding this theorem that $\mid f_{k}(x)-$ $f_{k-1}(x) \mid<(n+1) r$, which yields $(3)$.

Fix $y \in X$ and define

$$
V_{y}=\bigcap_{x_{0} \in E(y)} p_{x_{0}}^{-1}(0,1] \backslash \bigcup_{x_{0} \in X \backslash E^{\prime}(y)} \overline{p_{x_{0}}^{-1}(0,1]} .
$$

It is clear that $V_{y}$ is a neighborhood of $y$ since $\left\{\overline{p_{x_{0}}^{-1}(0,1]}: x_{0} \in X\right\}$ is a locally finite closed cover of $X$. Now pick an arbitrary $x \in V_{y}$. Our aim is to estimate $\left|f_{k}(y)-f_{k}(x)\right|$. To this end, first observe that $E(x) \subseteq E^{\prime}(y)$. Hence if $x_{0} \in E(x)$, then

$$
x \in p_{x_{0}}^{-1}(0,1] \subseteq U_{x_{0}}, \quad y \in \overline{p_{x_{0}}^{-1}(0,1]} \subseteq U_{x_{0}},
$$

and hence by (iii),

$$
\left|g_{x_{0}}(x)-g_{x_{0}}(y)\right|<2 \delta .
$$

Observe that for $x_{0} \in E(x)$ we have $g_{x_{0}}(y)-g_{x_{0}}(x) \in F(y)-F(x)$ and $\operatorname{dim}(F(y)-F(x)) \leq 2 n$. Hence again by the remark preceding this theorem,

$$
\left|\sum_{x_{0} \in E(x)} p_{x_{0}}(x)\left(g_{x_{0}}(y)-g_{x_{0}}(x)\right)\right| \leq(2 n+1) \cdot 2 \delta .
$$

Let $V_{y}^{k}$ be a neighborhood of $y$ that is contained in $V_{y}$ such that for every $x \in V_{y}^{k}$,

$$
\left|\sum_{x_{0} \in E^{\prime}(y)}\left(p_{x_{0}}(x)-p_{x_{0}}(y)\right) g_{x_{0}}(y)\right|<\frac{1}{2^{k+1}}
$$


We claim that $V_{y}^{k}$ has the properties required in (2). To prove this, let $x \in V_{y}^{k}$ be arbitrary. By (v), (vi) and $E(x) \subseteq E^{\prime}(y)$ (hence $p_{x_{0}}(x)=0$ if $\left.x_{0} \in E^{\prime}(y) \backslash E(x)\right)$ we clearly have

$$
\begin{aligned}
\left|f_{k}(y)-f_{k}(x)\right|= & \left|\sum_{x_{0} \in E^{\prime}(y)} p_{x_{0}}(y) g_{x_{0}}(y)-\sum_{x_{0} \in E(x)} p_{x_{0}}(x) g_{x_{0}}(x)\right| \\
\leq & \left|\sum_{x_{0} \in E^{\prime}(y)} p_{x_{0}}(y) g_{x_{0}}(y)-\sum_{x_{0} \in E^{\prime}(y)} p_{x_{0}}(x) g_{x_{0}}(y)\right| \\
& +\left|\sum_{x_{0} \in E(x)} p_{x_{0}}(x) g_{x_{0}}(y)-\sum_{x_{0} \in E(x)} p_{x_{0}}(x) g_{x_{0}}(x)\right| \\
\leq & \frac{1}{2^{k+1}}+(2 n+1) \cdot 2 \delta \leq \frac{1}{2^{k}} .
\end{aligned}
$$

Hence this completes the proof of the special case. To prove the general case from the special case, simply apply Remark 3.1.

The usual way of obtaining a continuous selection is to take the limit of a uniformly convergent sequence of (continuous) near-selections. In the proof of Theorem 4.1, a continuous selection was constructed as the limit of a sequence of possibly discontinuous selections. The other places that we found where a similar method was used are: Curtis [12], Michael [25], and Toruńczyk [5, Prop. 7.1 on p. 87].

Repovš and Semenov recently observed in [27, Theorem 1.6(2)] that the condition that each $F(x)$ is closed in Theorem 4.1 is redundant.

Let $Y$ be a convex subset of a metric linear space $(E, d)$. We say that $Y$ has the (finite-dimensional) selection property if, for every space $X$, every LSC-function $F: X \Rightarrow Y$ with (finite-dimensional) compact and convex values has a continuous selection.

We say that $Y$ has the (finite-dimensional) near-selection property if, for every space $X$, every LSC-function $F: X \Rightarrow Y$ with (finite-dimensional) compact and convex values has, for every $\varepsilon>0$, a continuous $\varepsilon$-near-selection. Sometimes near-selections exist and are useful in situations where continuous selections may not exist. See, e.g., Haver [21] for examples of such situations. For comments on the "hereditariness" of the (finite-dimensional) near-selection property, see $\S 6$.

We now come to the second main result in this paper.

THEOREM 4.2. Let $Y$ be a convex subset of a metric linear space E. Then $Y$ is an AR if and only if $Y$ has the finite-dimensional near-selection property.

Proof. Assume first that $Y$ is an AR. Let $F: X \Rightarrow Y$ be an LSC-function such that for every $x \in X, F(x)$ is a finite-dimensional convex compactum. 
Fix $\varepsilon>0$, and, for every $n \in \mathbb{N}$, put

$$
X_{n}=\{x \in X: \operatorname{dim} F(x) \leq n-1\} .
$$

Observe that $\bigcup_{n=1}^{\infty} X_{n}=X$, and every $X_{n}$ is closed in $X$ by Lemma 3.3. By Theorem 4.1, there exists a continuous selection $f_{n}: X_{n} \rightarrow E$ of $F \mid X_{n}$. Clearly, $f_{n}\left(X_{n}\right) \subseteq Y$. As $Y$ is an AR, we can extend $f_{n}$ to a map $\bar{f}_{n}: X \rightarrow Y$. Using Lemma 3.4, we can find an open set $U_{n}$ of $X$ such that $f_{n} \mid U_{n}$ is an $\varepsilon / 2^{n}$-near-selection of $F \mid U_{n}$. Now Lemma 3.5 does the job.

Conversely, let $f: A \rightarrow Y$ be a map of a closed subset $A$ of a space $X$. Let $\mathcal{U}$ be a locally finite open cover of $X \backslash A$ so that there exists for every $U \in \mathcal{U}$ an element $a_{U} \in A$ with $d\left(x, a_{U}\right) \leq 2 d(x, A)$ for all $x \in U$ (so $\mathcal{U}$ is a Dugundji cover of $X \backslash A$, see [26, Lemma 1.4.12]). Define $F: X \Rightarrow Y$ by

$$
F(x)= \begin{cases}\{f(x)\} & \text { for } x \in A, \\ \operatorname{conv}\left\{f\left(a_{U}\right): x \in U \in \mathcal{U}\right\} & \text { otherwise. }\end{cases}
$$

It is easy to see that $F$ is LSC ([26, Theorem 1.4.13]). Hence, there exists for every $\varepsilon>0$ a continuous function $\bar{f}_{\varepsilon}: X \rightarrow Y$ such that $d\left(\bar{f}_{\varepsilon}(x), F(x)\right)<\varepsilon$ for every $x \in A$. Since $Y$ is locally equiconnected, $f$ extends to a map $\bar{f}: X \rightarrow Y$ by Dobrowolski [13, Lemma 1].

So by Lemma 3.6 we get:

Corollary 4.3. Let the convex subset $Y$ of the metric linear space $E$ be an $\mathrm{AR}$ and $F: X \Rightarrow Y$ an LSC-function with compact finite-dimensional convex values. Then for every continuous $\varepsilon: X \rightarrow(0,1)$ there exists a continuous $f: X \rightarrow Y$ such that $d(f(x), F(x))<\varepsilon(x)$ for every $x \in X$.

5. Limitations in generalizing the results. In [9], Cauty proved that there is a certain separable metric linear space $E$ which is an AR that contains a closed linear subspace $L$ which is not an AR. In this section we will use this example among other things to illustrate the sharpness of our results. In the next section we will state some open problems and make some comments which we hope are illuminating.

(A) The dimensional restriction in Theorem 4.1. It is natural to ask whether the dimensional restriction in Theorem 4.1 can be weakened to: $\operatorname{dim} F(x)<\infty$ for all $x \in X$. We will show in Example 5.2 below that this cannot be done.

Let us say that a function $f: X \rightarrow Y$ is a $\sigma$-continuous selection for the function $F: X \Rightarrow Y$ provided that $f$ is a selection for $F$ and $X$ can be written as $\bigcup_{n=1}^{\infty} X_{n}$ such that, for each $n, X_{n}$ is closed in $X$ and $f \mid X_{n}$ is continuous (cf. Coban [10]). We will first show that certain maps admit $\sigma$-continuous selections. 
Lemma 5.1. Let $E$ be a metric linear space. If $F: X \Rightarrow E$ is an LSCfunction with closed and convex values and $\operatorname{dim} F(x)<\infty$ for every $x \in X$, then $F$ admits a $\sigma$-continuous selection.

Proof. For every $n$, put

$$
X_{n}=\{x \in X: \operatorname{dim} F(x) \leq n-1\} .
$$

Then every $X_{n}$ is closed by Lemma 3.3 , and $\bigcup_{n=1}^{\infty} X_{n}=X$. Now first observe that by Theorem 4.1 the restriction $F_{1}=F \mid X_{1}$ admits a continuous selection $f_{1}: X_{1} \rightarrow Y$. Define $F_{2}: X_{2} \Rightarrow Y$ by letting $F_{2}(x)=f_{1}(x)$ for $x \in X_{1}$, and $F_{2}(x)=F(x)$ otherwise. Since $F_{2}$ is LSC, again by Theorem 4.1, there exists a continuous selection $f_{2}: X_{2} \rightarrow Y$ for which necessarily $f_{2}(x)=f_{1}(x)$, $x \in X_{1}$. Proceeding inductively, we arrive at a sequence of maps $f_{n}: X_{n} \rightarrow Y$ that are continuous selections of $F \mid X_{n}$. Clearly, the function $f$ defined as the pointwise limit of the sequence $\left(f_{n}\right)_{n}$ is the desired $\sigma$-continuous selection.

One would hope that the construction in the proof of Lemma 5.1 would lead in certain cases to a global selection that is continuous. But this is not the case in general, as the following example shows, even if the domain of the function is as nice as the Hilbert cube $Q$. In fact, the example demonstrates much more. It shows that no choice of continuous partial selections results in a continuous limit function.

EXAMPLE 5.2. There are a metric linear space $E$ and an LSC-function $F$ from $Q$ to $E$ with finite-dimensional compact convex values such that $E$ contains a tower of closed subsets $\left\{E_{n}\right\}_{n}$ with the following properties:

(1) $Q=\bigcup_{n=1}^{\infty} Q_{n}$, where $Q_{n}=F^{\Leftarrow}\left(E_{n}\right)$,

(2) for every $n, F \mid Q_{n}: Q_{n} \Rightarrow E$ admits a continuous selection,

(3) if for every $n, f_{n}: Q_{n} \rightarrow E$ is a selection of $F \mid Q_{n}$ such that $f=$ $\lim _{n \rightarrow \infty} f_{n}: Q \rightarrow E$ is a well defined function, then $f$ is not continuous.

Proof. Consider the Cauty space E. In [9] it was proved that $E$ is the linear span of a linearly independent compact set (this will also be used in $\S 6$ ), hence $E$ is $\sigma$-compact. Consequently, it follows from Cauty's construction, or by applying results from Dobrowolski [13] or van der Bijl and van Mill [7], that there exists a compact subspace $K$ of $E$ such that the identity mapping on $K$ cannot be approximated arbitrarily closely by maps into finite-dimensional linear subspaces of $E$. We may think of $K$ as being a subspace of $Q$ as well. We claim that the identity mapping $1_{K}$ on $K$ cannot be extended to a continuous function $Q \rightarrow E$. For assume that this is not true, i.e., there is a continuous function $\alpha: Q \rightarrow E$ which restricts to the identity on $K$. It is clear that $\alpha$ can be approximated arbitrarily closely by maps of the form $\beta_{n} \circ \pi_{n}$, where $\pi_{n}: Q \rightarrow \mathbb{I}^{n}$ is the projection, and $\beta_{n}: \mathbb{I}^{n} \rightarrow E$ is continuous. But every continuous map from a finite-dimensional compact 
space into a metric linear space $E$ can be approximated arbitrarily closely by continuous maps into finite-dimensional linear subspaces of $E$. (This is well known: see, e.g., [6, Lemma 2.1].) So this clearly yields the desired contradiction.

Let $A=\left\{a_{n}: n \in \mathbb{N}\right\}$ be a countable dense subset of $K$. For every $n$, we put

$$
E_{n}=K \cup \operatorname{span}\left\{a_{1}, \ldots, a_{n}\right\}
$$

Let $f: K \rightarrow K$ denote the identity mapping. We now define $F: Q \Rightarrow E$ as in the proof of Theorem 4.2 with $a_{U} \in K$ for every $U \in \mathcal{U}$. It is clear that (1) holds, and (2) follows from Theorem 4.1. In addition, (3) holds as well, since if there existed a sequence of selections with continuous limit, then we would be able to extend the identity mapping on $K$ to a continuous function $Q \rightarrow E$.

The best result known to us that does not put dimensional restrictions on $F$ is Proposition 5.3 below. Variations of this result are well known. For completeness' sake we will sketch a proof that is both standard and direct.

Proposition 5.3. Let $E$ be a metric linear space. Then, for every locally finite-dimensional space $X$, every LSC-function $F: X \Rightarrow E$ with complete convex values has a continuous selection.

Proof. It is clear that by Remark 3.1 we may assume without loss of generality that $X$ is finite-dimensional. Hence the theorem follows from the complicated Theorem 1.2 in Michael [24]. For a direct proof, one can modify the simpler arguments in Michael [23], or observe that we can simply refine all open covers of the domain of our function by open covers of a fixed bounded order $n$. This will have the effect that the convex combinations that we have to take in our metric linear space will be combinations of at most $n$ vectors, which means that we can control distances in precisely the same way as in the proof of Theorem 4.2. The details of checking this are left to the reader.

REMARK 5.4. As an illustration of how Proposition 5.3 can be applied, let us consider a metric linear space $E$ with a complete linear subspace $E^{\prime}$. It is well known that if $E^{\prime}$ is finite-dimensional, then $E$ and $E / E^{\prime} \times E^{\prime}$ are homeomorphic (but not necessarily isomorphic). To get this from Proposition 5.3, simply observe that the function $F_{E^{\prime}}: E / E^{\prime} \Rightarrow E$ defined by $F([x])=x+E^{\prime}$ has a continuous selection. This suffices for the proof (see, e.g., [5, Corollary 7.1 on p. 86]). This result by the way is not best possible. It follows from Michael [25, Corollary 7.3] that for $E^{\prime}$, besides completeness, one only needs local convexity. In (the construction of) Example 5.8 below we will see that the function $F_{E^{\prime}}: E / E^{\prime} \Rightarrow E$ in general need not have a continuous selection. 
(B) Near-selections without dimensional restrictions. The following result on near-selections does not impose any dimensional restrictions on $F$. Recall that a space $X$ is a $C$-space if for every sequence $\mathcal{A}_{1}, \mathcal{A}_{2}, \ldots$ of open covers of $X$ there are pairwise disjoint open families $\mathcal{B}_{1}, \mathcal{B}_{2}, \ldots$ in $X$ such that $\mathcal{B}_{i}$ refines $\mathcal{A}_{i}$ for every $i$, and $\bigcup_{i=1}^{\infty} \mathcal{B}_{i}$ covers $X$. It is not difficult to prove that a countable-dimensional space is a $\mathrm{C}$-space.

Proposition 5.5. Let $Y$ be a convex subset of a metric linear space $E$. If $X$ is a $C$-space, then for every LSC-function $F: X \Rightarrow Y$ with convex values, and every continuous $\varepsilon: X \rightarrow(0,1)$, there exists a map $f: X \rightarrow Y$ such that $d(f(x), F(x))<\varepsilon(x)$ for every $x \in X$.

Proof. Let $\varepsilon>0$. We will first prove that $F$ admits an $\varepsilon$-near-selection. By assumption, for each $n \in \mathbb{N}$, there exists a disjoint open family $\mathcal{U}_{n}$ refining the cover $\left\{F^{\digamma}\left(B\left(y, \varepsilon / 2^{n+1}\right)\right): y \in Y\right\}$ such that $\bigcup_{n=1}^{\infty} \mathcal{U}_{n}$ covers $X$. Fix $n \in \mathbb{N}$. For every $U \in \mathcal{U}_{n}$ pick an element $y_{U} \in Y$ such that $U \subseteq B\left(y_{U}, \varepsilon / 2^{n}\right)$. The function $f_{n}: \bigcup \mathcal{U}_{n} \rightarrow Y$ defined by

$$
f_{n}(x)=y_{U} \Leftrightarrow x \in U
$$

is evidently an $\varepsilon / 2^{n+1}$-near-selection for $F \mid \bigcup \mathcal{U}_{n}$. Apply Lemma 3.5 to conclude that $F$ admits an $\varepsilon$-near-selection. To get the general result, now apply Lemma 3.6.

REMARK 5.6. We are indebted to the referee for pointing out that Proposition 5.5 is a consequence of complicated and much more general results in Uspenskiı [33]. (Let us note that results in [33] yield the assertion of Proposition 5.5 if the values $F(x)$ are merely star-shaped. This is because, for $\varepsilon>0$, $B(F(x), \varepsilon)$ is star-shaped (hence, contractible) provided $F(x)$ is, and, for every LSC-function $F: X \Rightarrow Y$, the set $\{(x, y) \in X \times Y: y \in B(F(x), \varepsilon)\}$ is open in $X \times Y$.) Since our proof is completely trivial, we decided to include it. Let us also remark that Proposition 5.5 cannot be extended beyond C-spaces (see Example 6.6).

REMARK 5.7. An immediate consequence of Proposition 5.5, together with the argument of Theorem 4.2, is that every convex subset of a metric linear space is an absolute extensor for the class of C-spaces. This is wellknown, and stronger results are available in the literature (cf. Addis and Gresham [1, Theorem 4.1], Ancel [2, Corollary C.5.10]).

This implies that if $Y$ is both a convex subset of a metric linear space and a $\mathrm{C}$-space, then it is an AR. This is folklore and for the convenience of the reader we sketch the proof. Indeed, let $Z$ containing $Y$ as a closed subspace be such that $Z$ is an ANR and $Z \backslash Y$ is a polytope (hence is countabledimensional). (For details, see, e.g., Hu [22, p. 53]. It is not explicitly stated there that the resulting space is an ANR. That this is true is well known and follows easily from the characterization theorem in [22, p. 122]. See 
also Borsuk [8, p. 107].) Hence $Z$ is a C-space by Addis and Gresham [1, Theorem 2.1], and we can apply Remark 5.7 to conclude that $Y$ is a retract of $Z$, hence $Y$ is an ANR, and so an AR by contractibility. We do not know of a more elementary proof that convex C-spaces (in particular, convex subsets of $\aleph_{0}$-dimensional metric linear spaces) are AR's.

The following example shows that Proposition 5.3 does not hold in case $X$ is an arbitrary strongly countable-dimensional space (or even, when $X$ is a countable union of finite-dimensional compacta).

EXAMPLE 5.8. There are a metric linear space $E$ and a linear subspace $L$ of $E$ such that $L$ is complete and $X=E / L$ is $\aleph_{0}$-dimensional (hence strongly countable-dimensional) while the standard LSC-function $F=F_{L}: X \Rightarrow E$ defined by $F([x])=x+L$ has the following properties:

(1) $F$ does not admit a continuous selection,

(2) for every continuous $\varepsilon: X \rightarrow(0,1)$, there exists a continuous function $f: X \rightarrow L$ such that $d(f(x), F(x))<\varepsilon(x)$ for every $x \in X$.

Proof. Dobrowolski and Kalton [14] proved that Cauty's construction in [9] can be used to construct an example of a complete separable metric linear space $E^{\prime}$ which is an AR, having a closed linear subspace $L$ which is not an AR (recall that the original Cauty space is $\sigma$-compact). Let $A$ be a countable dense subset of $E^{\prime}$, and put $E=\operatorname{span}(L \cup A)$. Then $E$ is a dense linear subspace of $E^{\prime}$, whence $E$ is an AR because $E^{\prime}$ is (see $\S 2$ ). Then $X=E / L$ is an $\aleph_{0}$-dimensional metric linear space and hence is strongly countabledimensional. Now if the standard LSC-function $F_{L}: X \Rightarrow E$ had a continuous selection, then $E$ would be homeomorphic to $X \times L$ (cf. [5, Corollary 7.1 on p. 86]), which would violate $L$ being a non-AR. This proves (1). For (2), apply Proposition 5.5.

Observe that the function $F$ in the proof of Example 5.8 is even continuous.

6. Remarks and open questions. As announced in $\S 5$, we will state here some open problems and make some further remarks that will put some of our results in their proper perspectives.

(A) Convex-hereditary Absolute Retracts and (near-) selections. It is trivial that the (finite-dimensional) selection property is hereditary with respect to convex subsets. As a consequence, every convex subset of a metric linear space with the finite-dimensional selection property is a "convex-hereditary Absolute Retract". This means that the finite-dimensional selection property is strictly stronger than the AR-property in metric linear spaces. Simply consider Cauty's linear space $E$ and its linear subspace $L$ that we used in $\S 5$, and apply Theorem 4.2. The near-selection property and the finite-dimensional 
near-selection property are not hereditary however, not even for closed linear subspaces. To see this, again consider the Cauty spaces $E$ and $L$ and again use the fact that $E$ is the linear span of a linearly independent compact set. In such a space every convex compactum is finite-dimensional (Dobrowolski and Mogilski [15, Example on p. 657]). But the near-selection property and the finite-dimensional near-selection property are hereditary on dense convex sets. The proof of this is similar to the standard proof that a dense linear subspace of a metric linear AR is an AR (for a sketch of that proof, see §2).

The only known convex subsets of metric linear spaces that are convexhereditary Absolute Retracts are the ones that are locally convex or are $\mathrm{C}$-spaces (among them, the convex subsets of $\aleph_{0}$-dimensional metric linear spaces); see Remark 5.7. Since every convex set with the finite-dimensional selection property is a convex-hereditary Absolute Retract, the following two questions are quite natural.

Question 6.1. Let $E$ be a metric linear space. Are the following statements about $E$ equivalent:

(a) $E$ is a convex-hereditary Absolute Retract.

(b) $E$ has the finite-dimensional selection property.

(c) $E$ has the selection property.

Question 6.2. Is the space $\ell^{p}, 0<p<1$, a convex-hereditary Absolute Retract?

It is an intriguing problem whether Theorem 4.1 can be extended to the case where the LSC-function $F$ has no dimensional restrictions but instead $E$ is $\aleph_{0}$-dimensional.

QUESTION 6.3. Let $E$ be an $\aleph_{0}$-dimensional metric linear space. If $F$ : $X \Rightarrow E$ is an LSC-function with complete and convex values, does $F$ admit a continuous selection? If not, does $E$ have the finite-dimensional selection property?

We even do not know the answer if $E$ is as simple as the space of all eventually zero sequences in $\ell^{p}$, where $0<p<1$. Actually, we do not know a single example of a nonlocally convex metric linear space $E$ that has the finite-dimensional selection property.

An interesting special case of Question 6.3 is the following:

Question 6.4. Let $E$ be an $\aleph_{0}$-dimensional metric linear space. If $X$ is a countable union of zero-dimensional subspaces, i.e., if $X$ is $\aleph_{0}$-dimensional, does every LSC $F: X \Rightarrow E$ with finite-dimensional compact convex values have a continuous selection? What if $X$ is equal to $\sigma=\{x \in Q:(\exists N \in$ $\left.\mathbb{N})(\forall n \geq N)\left(x_{n}=0\right)\right\}$ ? 
It follows from Uspenskij [33] (or Proposition 5.5) that a function $F$ such as in Question 6.4 has $\varepsilon$-near-selections for every $\varepsilon>0$. In fact, this even holds for functions $F$ for which the sets $F(x)$ are (not necessarily compact) star-shaped (see Remark 5.6).

(B) Nicely placed subspaces in metric linear AR's. We showed in Theorem 4.2 that a convex subset of a metric linear space is an AR if and only if it has the finite-dimensional near-selection property. This prompts the following central question.

Question 6.5. Let $E$ be a metric linear space. If $E$ is an AR, does $E$ have the near-selection property? What if $E=\ell^{p}$ and $0<p<1$ ?

In $\S 3$ we used Cauty's space to get a linear space for which the conclusion of the Michael Selection Theorem fails. A variation of this example will be constructed in Example 6.6 below. Consider the natural LSC function $F_{E^{\prime}}: E / E^{\prime} \Rightarrow E$ for a closed linear subspace $E^{\prime}$ of a metric linear space $E \in \mathrm{AR}$. Then if $F_{E^{\prime}}$ admits a continuous selection it follows that both $E^{\prime}$ and $E / E^{\prime}$ are AR's (see Remark 5.4). If, however, $F_{E^{\prime}}$ has merely an $\varepsilon$-near-selection for every $\varepsilon>0$ then $E / E^{\prime}$ is an AR, as stated in Bessaga and Dobrowolski [4, p. 40], while $E^{\prime}$ may not be an AR (see Example 5.8). Let us say that a closed linear subspace $E^{\prime}$ of a metric linear space $E$ is nicely placed in $E$ provided that $F_{E^{\prime}}$ has an $\varepsilon$-near-selection for every $\varepsilon>0$.

EXAMPLE 6.6. There exists a metric linear space $E$ homeomorphic to $\ell^{2}$ and a closed linear subspace $E^{\prime}$ of $E$ which is not nicely placed in $E$ (hence the natural function $F_{E^{\prime}}: E / E^{\prime} \Rightarrow E$ does not have an $\varepsilon$-near-selection for some $\varepsilon>0)$.

Proof. By a result of Terry [31], there is a so-called couniversal space for separable metric linear spaces, that is, a separable complete metric linear space $Z$ such that for every separable complete metric linear space $E$ there is a closed linear subspace $Z^{\prime}$ of $Z$ such that $Z / Z^{\prime}$ and $E$ are isomorphic. Interestingly, $Z$ is homeomorphic to $\ell^{2}$. Applying this for the completion $\widehat{L}$ of the Cauty space $L$ (see $\S 5$ ), we get by the just quoted observation of Bessaga and Dobrowolski a closed linear subspace $Z^{\prime}$ of $Z$ such that $F_{Z^{\prime}}: \widehat{L}=$ $Z / Z^{\prime} \Rightarrow Z$ does not have an $\varepsilon$-near-selection for some $\varepsilon>0$.

REMARK 6.7. Let $E^{\prime}$ be a closed linear subspace of a metric linear space $E$. If $E$ is separable then $E^{\prime}$ can be enlarged to a dense linear subspace $E^{\prime \prime}$ of $E$ so that $E^{\prime}$ is nicely placed in $E^{\prime \prime}$. By Proposition 5.5, any dense $E^{\prime \prime}$ for which $E^{\prime \prime} / E^{\prime}$ is $\aleph_{0}$-dimensional will do.

By applying Remark 6.7, the linear subspace $Z^{\prime}$ of $Z$ from the construction of Example 6.6 can be enlarged to a dense linear subspace $Z^{\prime \prime}$ in which $Z^{\prime}$ is nicely placed; yet, $Z^{\prime}$ is not nicely placed in $Z$. 
For the reader's convenience, we provide a simple proof of the Bessaga and Dobrowolski observation, which we generalize to convex sets. For a convex set $C$ in a metric linear space $E$, let $X=\kappa(C)$, where $\kappa: E \rightarrow E / E^{\prime}$ is the natural quotient map. Define $F^{C}: X \rightarrow C$ by $F^{C}([x])=\left(x+E^{\prime}\right) \cap C$. Then we have:

Lemma 6.8. Assume that $F^{C}$ has an $\varepsilon$-near-selection for every $\varepsilon>0$. Then if $C$ is an $\mathrm{AR}$, so is $X$.

Proof. Simply observe that $\kappa \mid C: C \rightarrow X$ admits $\varepsilon$-right inverses for every $\varepsilon>0$. Hence we can apply [22, Theorem 5.3] to conclude that $X$ is an ANR and, therefore, an AR by contractibility.

For $E=C$, the assumption in Lemma 6.8 simply says that $E^{\prime}$ is nicely placed in $E$. It should be noted, however, that unlike $F_{E^{\prime}}$, the function $F^{C}$ may not be LSC, even in the case when $C$ is a closed cone in a 3-dimensional space $E$ (see Bauschke and Borwein [3, Example 2.3]). So, constructing $\varepsilon$ near-selections for $F^{C}$ may be a very difficult task.

Question 6.9. Are all closed linear subspaces $E^{\prime}$ of $\ell^{p}, 0<p<1$, nicely placed in $\ell^{p}$ ?

(C) Near-selections with stronger properties. Let $Y$ be a convex subset of a metric linear space $E$, and let $F: X \Rightarrow Y$ be LSC with convex values. We consider the following statements about $F$ :

(I) For each $\varepsilon>0$ there is a continuous function $f: X \rightarrow Y$ such that $d(f(x), F(x))<\varepsilon$ for every $x \in X$.

(II) For each continuous $\varepsilon: X \rightarrow(0,1)$ there is a continuous function $f: X \rightarrow Y$ such that $d(f(x), F(x))<\varepsilon(x)$ for every $x \in X$.

(III) For every open cover $\mathcal{U}$ of $Y$ there exists a continuous function $f: X \rightarrow Y$ such that for every $x \in X$ there exists an element $U \in \mathcal{U}$ such that $f(x) \in U$ and $U \cap F(x) \neq \emptyset$.

(IV) For every continuous function $\delta: Y \rightarrow(0,1)$ there is a continuous function $f: X \rightarrow Y$ such that $d(f(x), F(x))<\delta(f(x))$ for every $x \in X$.

The reader can easily verify that (III) $\Leftrightarrow(\mathrm{IV}) \Rightarrow(\mathrm{I}) \Leftrightarrow(\mathrm{II})$ (cf. Lemma 3.6). This raises the interesting problem whether all four properties are equivalent even if the values of $F$ are not only convex but also compact. This does not seem to be trivial. Simply observe that (III) is "topological", but (I) is not.

\section{References}

[1] D. F. Addis and J. H. Gresham, A class of infinite-dimensional spaces. I. Dimension theory and Alexandroff's problem, Fund. Math. 101 (1978), 195-205. 
[2] F. D. Ancel, The role of countable dimensionality in the theory of cell-like relations, Trans. Amer. Math. Soc. 287 (1985), 1-40.

[3] H. H. Bauschke and J. M. Borwein, Conical open mapping theorems and regularity, preprint, 2003.

[4] C. Bessaga and T. Dobrowolski, Some open problems on the border of infinitedimensional topology and functional analysis, in: Proc. Internat. Conf. on Geometric Topology, K. Borsuk and A. Kirkor (eds.), PWN-Polish Sci. Publ., Warszawa, 1980, $39-42$.

[5] C. Bessaga and A. Pełczyński, Selected Topics in Infinite-Dimensional Topology, Monogr. Mat. 58, PWN-Polish Sci. Publ., Warszawa, 1975.

[6] J. van der Bijl, T. Dobrowolski, K. P. Hart, and J. van Mill, Admissibility, homeomorphism extension and the AR-property in topological linear spaces, Topology Appl. 48 (1992), 63-81.

[7] J. van der Bijl and J. van Mill, Linear spaces, Absolute Retracts, and the Compact Extension Property, Proc. Amer. Math. Soc. 104 (1988), 942-952.

[8] K. Borsuk, Theory of Retracts, Monogr. Mat. 44, PWN-Polish Sci. Publ., Warszawa, 1967.

[9] R. Cauty, Un espace métrique linéaire qui n'est pas un rétracte absolu, Fund. Math. 146 (1994), 85-99.

[10] M. M. Čoban, General theorems on selections and their applications, Serdica 4 (1978), 74-90.

[11] H. H. Corson and J. Lindenstrauss, Continuous selections with nonmetrizable range, Trans. Amer. Math. Soc. 121 (1966), 492-504.

[12] D. W. Curtis, Application of a selection theorem to hyperspace contractibility, Canad. J. Math. 37 (1985), 747-759.

[13] T. Dobrowolski, On extending mappings into nonlocally convex linear metric spaces, Proc. Amer. Math. Soc. 93 (1985), 555-560.

[14] T. Dobrowolski and N. J. Kalton, Extracting more info on Cauty's space, preprint, 2004 .

[15] T. Dobrowolski and J. Mogilski, Sigma-compact locally convex metric linear spaces universal for compacta are homeomorphic, Proc. Amer. Math. Soc. 85 (1982), 653658.

[16] - - - Certain sequence and function spaces homeomorphic to the countable product of $l_{f}^{2}$, J. London Math. Soc. (2) 45 (1992), 566-576.

[17] T. Dobrowolski and H. Toruńczyk, Separable complete ANRs admitting a group structure are Hilbert manifolds, Topology Appl. 12 (1981), 229-235.

[18] J. Dugundji, An extension of Tietze's theorem, Pacific J. Math. 1 (1951), 353-367.

[19] -, Topology, Allyn and Bacon, Boston, 1966.

[20] W. E. Haver, Locally contractible spaces that are absolute neighborhood retracts, Proc. Amer. Math. Soc. 40 (1973), 280-284.

[21] —, A near-selection theorem, General Topology Appl. 9 (1978), 117-124.

[22] S. T. Hu, Theory of Retracts, Wayne State Univ. Press, Detroit, 1965.

[23] E. A. Michael, Continuous selections. I, Ann. of Math. 63 (1956), 361-382.

[24] -, Continuous selections II, ibid. 64 (1956), 562-580.

[25] - Convex structures and continuous selections, Canad. J. Math. 11 (1959), 556575 .

[26] J. van Mill, Infinite-Dimensional Topology: Prerequisites and Introduction, NorthHolland, Amsterdam, 1989.

[27] D. Repovš and P. Semenov, On closedness assumptions in selection theory, preprint, 2005 . 
[28] D. Repovš and P. V. Semenov, Continuous Selections of Multivalued Mappings, Math. Appl. 455, Kluwer, Dordrecht, 1998.

[29] W. Rudin, Functional Analysis, McGraw-Hill, New York, 1991.

[30] R. Schneider, Convex Bodies: the Brunn-Minkowski Theory, Encyclopedia Math. Appl. 44, Cambridge Univ. Press, Cambridge, 1993.

[31] W. E. Terry, F-spaces universal with respect to linear codimension, Proc. Amer. Math. Soc. 63 (1977), 59-65.

[32] H. Toruńczyk, Concerning locally homotopy negligible sets and characterizations of $\ell_{2}$-manifolds, Fund. Math. 101 (1978), 93-110.

[33] V. V. Uspenskij, A selection theorem for C-spaces, Topology Appl. 85 (1998), 351374 .

Department of Mathematics

Pittsburg State University

Pittsburg, KS 66762, U.S.A.

E-mail: tdobrowo@mail.pittstate.edu
Faculteit Exacte Wetenschappen Afdeling Wiskunde Vrije Universiteit De Boelelaan 1081A 1081 HV Amsterdam, The Netherlands E-mail: vanmill@cs.vu.nl

Received 15 November 2004; in revised form 5 October 2006 